\title{
Spectral Properties of Many-body Schrödinger Operators with Dilatation-analytic Interactions
}

\author{
E. BALSLEV \\ Department of Mathematics, SUNY at Buffalo, New York,USA \\ J. M. COMBES \\ Centre de Physique Théorique - C.N.R.S., Marseille, France
}

Received March 2, 1971

\begin{abstract}
Quantum mechanical $N$-body systems with dilatation analytic interactions are investigated. Absence of continuous singular part for the Hamiltonians is proved together with the existence of an absolutely continuous part having spectrum $\left[\lambda_{e}, \infty\right)$, where $\lambda_{e}$ is the lowest many body threshold of the system. In the complement of the set of thresholds the point spectrum is discrete; corresponding bound state wave-functions are analytic with respect to the dilatation group.
\end{abstract}

\section{Introduction}

Some important progress has been made during the last years in the mathematical analysis of multiparticle quantum scattering systems. The main results concern non-relativistic three or four particle systems with smooth short-range forces (see e.g. [1]). However no definite step has been made toward a general proof of some fundamental problems such as asymptotic completeness except for repulsive potentials [1,2] or locally for general multichannel systems [3]. Another troublesome situation concerns the inclusion of more general forces, Coulomb like or electromagnetic, in multiparticle formalisms. First steps in this direction have been made by proving existence of generalized wave-operators for multichannel systems with potentials $g / r^{\beta},<\beta \leqq 1([4,5])$; these improvements on traditional time-dependent methods should support the future extension to multiparticle systems of recent formulations of scattering theory with such general forces (see e.g. [6]). It has to be expected that further progress in these domains can be made from a systematic study of the spectral properties of Schrödinger Hamiltonians. For example the experience of the $N$ particle problem with short-range potentials strongly suggests that fundamental ingredients of asymptotic completeness are the absence of continuous singular spectrum and the 
discreteness of bound-states. The first requirement guarantees absence of pathological states which are neither bound-states nor scattering states in the sense that the probability that particles remain in a bounded domain of configuration space does not tend to zero as the time increases. The second requirement is needed for a correct space-time behavior of wave-packets in multichannel scattering; furthermore it has obvious consequences for the analytic structure of the $S$-matrix. Both problems have been solved in the one body case [7] with the help of an analyticity assumption on potentials with respect to the dilatation group. Such a condition is rather weak and it requires neither short-range, locality nor respulsivity of forces, allowing in particular some electromagnetic fields. We investigate here multiparticle systems with such forces using Weinberg equations; our main tool is the analyticity of its solutions with respect to the dilatation parameter. From this one can deduce the existence of a meromorphy domain for expectation values of the resolvent allowing their analytic continuation through the whole set of channel cuts. This implies absence of continuous singular spectrum and the correct spectrum $\left[\lambda_{e}, \infty\right)$ for the absolutely continuous part of the Hamiltonians, where $\lambda_{e}$ is the lowest many-body threshold of the system. The intersection of the discrete spectrum with the complement of the set of thresholds is a bounded set consisting of isolated eigenvalues with finite multiplicities.

Let $H=H_{0}+V, V=\sum_{\substack{\alpha=(i j) \\ i \neq j}} V_{\alpha}$, be the Schrödinger operator of the system $M$ of $n$ particles with two-body interactions $V_{\alpha}$. We have separated the center-of-mass of $M$, so the space is $\mathscr{H}=L^{2}\left(R^{3 n-1}\right)$. The operator $H_{0}$ representing the kinetic energy is obtained from the classical kinetic energy $\sum_{i=1}^{n} \frac{\left|\bar{p}_{i}\right|^{2}}{2 m_{i}}$ by the substitution $\bar{p}_{i} \rightarrow \frac{\hbar}{i} \nabla_{i}$ and 3 of the variables are eliminated through the equation $\sum_{i=1}^{n} \bar{p}_{i}=0$.

We refer to other papers on multiparticle systems (see e.g. [1,3]) for kinematical details. We just mention some basic facts which are needed in this paper. To each cluster decomposition $D=\left\{C_{1}, C_{2}, \ldots C_{k}\right\}$, $C_{i} \cap C_{j}=\Phi, i \neq j, \cup C_{i}=M$, there corresponds a tensor decomposition

$$
\mathscr{H}=\mathscr{H}^{C_{1}} \otimes \mathscr{H}^{C_{2}} \otimes \cdots \otimes \mathscr{H}^{C_{k}} \otimes \mathscr{H}^{C_{1}, C_{2}}, \ldots C_{k}
$$

where $\mathscr{H}^{C}$ is the state space for particles belonging to $C$ (center of mass separated) and $\mathscr{H}^{\left(C_{1}, C_{2}, \ldots, C_{k}\right)}$ is the state space for the centers of mass of clusters. The operator $H_{0}$ splits into a sum over clusters of internal

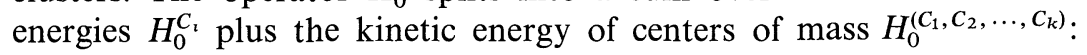

$$
H_{0}=H_{0}^{C_{1}}+H_{0}^{C_{2}}=\cdots+H_{0}^{\left(C_{1}, C_{2}, \ldots, C_{k}\right)}
$$


where e.g. $H_{0}^{C_{1}}$ has to be understood as an operator on $\mathscr{H}$, that is the tensor product of the kinetic energy operator on $\mathscr{H}^{C_{1}}$ and the identity operator on the Hilbert space corresponding to other degrees of freedom. We adopt the convention, unless explicitly needed, to denote in the same way an operator on one component of (1) or the corresponding operator on $\mathscr{H}$.

We assume that the interactions $V_{\alpha}, \alpha=(i, j), i \neq j$ are symmetric operators in $\mathscr{H}^{\alpha} \approx L^{2}\left(R^{3}\right)$ compact with respect to $\Delta$, i.e. the operator

$$
V_{\alpha}: \mathscr{D}(\Delta) \rightarrow L^{2}\left(R^{3}\right)
$$

is compact. Then $H$ is self-adjoint and $\mathscr{D}(H)=\mathscr{D}\left(H_{0}\right)$. In fact our hypothesis implies that the interactions $V_{\alpha}$ are $H_{0}-\varepsilon$ bounded (see e.g. [8], App. I).

Let $U(\theta), \theta \in R$, be the strongly continuous unitary representation on $\mathscr{H}$ of the dilatation group defined by

$$
(U(\theta) f)(\vec{r})=e^{\frac{3(n-1) \theta}{2}} f\left(e^{\theta} \vec{r}\right) .
$$

For each cluster decomposition $U(\theta)$ is a tensor product of the unitary representations of the dilatation group on the Hilbert spaces occuring in the decompositions (1).

We now make the second basic assumption about the interactions $V_{\alpha}$. Let

$$
V_{\alpha}(\theta)=U(\theta) V_{\alpha} U(\theta)^{-1}, \quad \theta \in R .
$$

The mapping $R \rightarrow V_{\alpha}(\theta)$ is a compact operator valued function on $R$. We assume that the function $V_{\alpha}(\theta)$ has an analytic extension to a domain $\mathcal{O}$, such that $\mathcal{O} \cap R \neq \Phi$, as a function on $\mathcal{O}$ whose values are bounded operators from $\mathscr{D}(\Delta)$ into $L^{2}\left(R^{3}\right)$. It follows (see $\left.[12,14]\right)$ that $V_{\alpha}(\theta)$ is a compact operator for every $\theta \in \mathcal{O}$. We notice that for $\operatorname{Im} \theta=\beta=$ constant, the operators $V_{\alpha}(\theta)$ are unitarily equivalent. Setting

$$
V_{\alpha, t}(\theta)=U(t) V_{\alpha}(\theta) U(t)^{-1}
$$

for every fixed $t \in R$, we obtain analytic functions $V_{\alpha, t}(\theta)$ on the domains $\mathcal{O}_{t}=\mathcal{O}+t$, such that $V_{\alpha, t_{1}}(\theta)=V_{\alpha, t_{2}}(\theta)$ for $\theta \in \mathcal{O}_{t_{1}} \cap \mathcal{O}_{t_{2}}$. The function $V_{\alpha}(\theta)$ defined by $V_{\alpha}(\theta)=V_{\alpha, t}(\theta)$ for any $t$ such that $\theta \in \mathcal{O}_{t}$ is analytic on the strip $\mathcal{O}+R$. Moreover, the function $\alpha^{*}(\bar{\theta})=\alpha(\theta)$ is an analytic continuation of $\alpha(\theta)$ into the domain symmetric to $\mathcal{O}$ with respect to the real axis $R$. Thus we can assume that $V_{\alpha}(\theta)$ is a compact operator valued function defined on a strip symmetric to the real axis, $\mathcal{O}=\mathcal{O}_{a}=\{z|| \operatorname{Im} z \mid<a\}$, where we assume $a \leqq \pi / 4$. Consider now the family of operators $H(\theta)$ 
defined for $\theta \in R$ by

$$
H(\theta)=U(\theta) H U(\theta)^{-1}=e^{-2 \theta} H_{0}+V(\theta) .
$$

Since $V(\theta)$ is $H_{0}-\varepsilon$-bounded, this family has a self-adjoint analytic extension to $\mathcal{O}$ defined by

$$
H(\theta)=e^{-2 \theta} H_{0}+V(\theta), \quad \theta \in \mathcal{O} .
$$

We shall now investigate the spectra of the operators $H(\theta), \theta \in \mathcal{O}_{a} \backslash R$ $=\{z|0<| \operatorname{Im} z \mid<a\}$, and then make the transition to real $\theta$. Since the operators $H(\alpha+i \beta),-\infty<\alpha<\infty$, are unitarily equivalent, the spectrum $\sigma(H(\theta))$ depends on $\beta$ only, $-a<\beta<a$.

For $\theta=\alpha+i \beta \in \mathcal{O}_{a}$ we introduce the following

Definition $\sigma_{\beta}=\sigma(H(\theta))$,

$\varrho_{\beta}=\varrho(H(\theta))$,

$\sigma_{i \beta}=$ the set of isolated points in $\sigma_{\beta}$,

$\sigma_{e \beta}=\sigma_{\beta} \backslash \sigma_{i \beta}$,

$\sigma_{p \beta}=$ the set of eigenvalues of $H(\theta)$,

$\sigma_{d \beta}=$ the set of isolated eigenvalues of $H(\theta)$ of finite multiplicity.

We omit the subscript $\beta$ for $\beta=0$, i.e. $\sigma=\sigma_{0}$ etc. We set $R(\theta, z)$ $=(H(\theta)-z)^{-1}$ for $z \in \varrho_{\beta}$.

For any set $S$ contained in the complex plane we write $S^{r}=S \cap R$ and $S^{\prime}$ denotes the set of accumulation points of $S$.

For $C \subset M$ we denote by $H^{C}(\theta)$ the operator defined as $H(\theta)$ for the system $C$, i.e.

$$
H^{C}(\theta)=e^{-2 \theta} H_{0}^{C}+V^{C}(\theta)
$$

where $H_{0}^{C}$ is the free Hamiltonian of $C$ relative to the center-of-mass of $C$ and $V^{C}(\theta)=\sum_{\substack{i, j \in C \\ i \neq j}} V_{i j}(\theta)$. We denote by $\sigma^{C}(\theta)$ the spectrum of $\sigma\left(H^{C}(\theta)\right)$ etc.

We denote by $\Sigma_{\beta}$ the set of all sums of isolated finite-dimensional eigenvalues of Hamiltonians of disjoint clusters of the system and the point 0 , i.e.

$$
\Sigma_{\beta}=\{0\} \underset{C_{1} \cup \cdots \cup C_{p} \leqq M}{\bigcup}\left(\sigma_{d \beta}^{C_{1}}+\cdots+\sigma_{d \beta}^{C_{p}}\right)
$$

(the trivial decomposition $M=M$ is not allowed. For $n=2, \Sigma_{\beta}=\{0\}$ ). Let

$$
\lambda_{0 \beta}=\inf _{\lambda \in \sigma_{\beta}^{r}} \lambda, \quad \lambda_{e \beta}=\inf _{\lambda \in \Sigma_{\beta}^{r}} \lambda .
$$

For $\lambda \in R, S_{\beta}(\lambda)$ is the sector of $C$ bounded by the half-lines $\left\{\lambda+R^{+}\right\}$and $\left\{\lambda+e^{-2 i \beta} R^{+}\right\}$. 
Now the basic qualitative properties of $\sigma_{\beta}, \beta \neq 0$, can be formulated in the following

Lemma 1. The spectrum $\sigma_{\beta}$ has the properties

(i) $\sigma_{e \beta}=\Sigma_{\beta}+e^{-2 i \beta} R^{+}$.

(ii) $\sigma_{\beta} \backslash \sigma_{\beta}^{r} \subset S_{\beta}\left(\lambda_{e \beta}\right)$.

(iii) $\sigma_{d \beta}=\sigma_{i \beta}$.

$\sigma_{e \beta}^{r}=\sum_{\beta}^{r}$ and $\sigma_{d \beta}^{r}$ are constant for $0<|\beta|<a, \sigma_{\beta}^{r}$ is bounded and $\sigma_{d \beta}^{r \prime} \subset \Sigma_{\beta}^{r}$.

(iv) For $|\varphi|>2|\beta|$ there exists $C(\varphi)>0$ such that for $0 \leqq \varrho<\infty$,

$$
\left\|\left(H(\theta)-\lambda_{0 \beta}+1-\varrho e^{i \varphi}\right)^{-1}\right\| \leqq C(\varphi) \varrho^{-1} .
$$

Briefly formulated the Lemma states that the spectrum of $H(\theta)$ consists of a system of parallel half-lines in the direction $e^{-2 i \beta}$ starting from the points of $\Sigma_{\beta}$ and a set of isolated, finite-dimensional eigenvalues, which can be divided into a bounded set of real eigenvalues accumulating at most at points of $\Sigma_{\beta}^{r}$ and a set of non-real, finite dimensional eigenvalues contained in the sector $S_{\beta}$. The resolvent $R(\theta, z)$ satisfies the estimate (iv).

Proof. We prove the Lemma by induction on $n$

1) $n=2$.

We have

$$
H(\theta)=e^{-2 \theta} H_{0}+V(\theta)=e^{-2 \theta}\left(H_{0}+e^{2 \theta} V(\theta)\right), \quad H_{0}=-\Delta .
$$

The essential spectrum of $H_{0}$ is $[0, \infty)$. Since the operator $e^{2 \theta} V(\theta)$ is $H_{0}$-compact, it follows that $\sigma_{e}\left(H_{0}+e^{2 \theta} \mathrm{v}(\theta)\right)=[0, \infty)$, and in the complement of $[0, \infty)$ the spectrum of $H_{0}+e^{2 \theta} V(\theta)$ contains only finitedimensional isolated eigenvalues of $H_{0}+e^{2 \theta} V(\theta)$ and complex conjugates of finite-dimensional isolated eigenvalues of $H_{0}+e^{2 \bar{\theta}} V(\bar{\theta})$ (see [13]). Hence

(i) $\sigma_{e \beta}=e^{-2 i \beta} R^{+}$

and in $\complement \sigma_{e \beta}$ the spectrum $\sigma_{\beta}$ consists of finite-dimensional isolated eigenvalues of $H(\theta)$ and complex conjugates of finite-dimensional eigenvalues of $H(\bar{\theta})$. Since the conjugate of an eigenvalue of $H(\theta)$ is an eigenvalue of $H(\theta)$ of the same multiplicity, we conclude that $\sigma_{i \beta}=\sigma_{d \beta}$. This argument presumes that the operator is local, but can be extended to the more general case where there exists an antiunitary operator $\mathscr{T}$ such that $\mathscr{T}^{2}=1$ and $H(\bar{\theta})=\mathscr{T} H(\theta) \mathscr{T}$.

It is immediate from (i) that $\sigma_{e \beta}^{r}=\{0\}$ for $0<|\beta|<a$. We shall now prove that $\sigma_{d \beta}^{r}$ is independent of $\beta$ as well as (ii) using the analyticity. Let $\theta_{0}=\alpha_{0}+i \beta_{0} \in \mathcal{O}, z \in \varrho_{\beta_{0}}$. Then since the relative norm of $H(\theta)-H\left(\theta_{0}\right)$ $: \mathscr{D}\left(H\left(\theta_{0}\right)\right) \rightarrow \mathscr{H}$, goes to 0 as $\theta \rightarrow \theta_{0}$ it follows that $z \in \varrho_{\beta}$ for $\left|\beta-\beta_{0}\right|<\varepsilon$. Suppose on the other hand that $\lambda_{0}$ is an isolated eigenvalue of $H\left(\theta_{0}\right)$. Then $\lambda_{0}$ is a multivalued analytic function of $\theta$ for $\left|\theta-\theta_{0}\right|<\delta$, and $\lambda_{0}$ is an 
isolated eigenvalue of $H\left(\alpha+i \beta_{0}\right)$ for all $\alpha$, hence $\lambda_{0}$ is an isolated eigenvalue of $H(\theta)$ for $\left|\beta-\beta_{0}\right|<\delta$. Thus the sets $\left\{\beta \in(-a, a) \mid \lambda \in \varrho_{\beta}\right\}$ and $\left\{\beta \in(-a, a) \mid \lambda \in \sigma_{d \beta}\right\}$ are both open. Let $\beta_{0}>0$ be fixed, $-a<\beta_{0}<a$, and let $z \notin S_{\beta_{0}}(0) \cup R$, i.e. $z \notin R, \arg z \notin\left[-2 \beta_{0}, 0\right]$. Then there is an interval $\left(-K_{1}, \beta_{0}+K_{2}\right), K_{1}, K_{2}>0$, such that $z \notin \sigma_{e \beta}$ for $\beta \in\left(-K_{1}, \beta_{0}+K_{2}\right)$, so either $z \in \varrho_{\beta}$ or $z \in \sigma_{d \beta}$.

Since $z \in \varrho_{0}$, and both the sets $\left\{\beta \in\left(-K_{1}, \beta_{0}+K_{2}\right) \mid z \in \varrho_{\beta}\right\}$ and $\left\{\beta \in\left(-K_{1}, \beta_{0}+K_{2}\right) \mid z \in \varrho_{d \beta}\right\}$ are open, it follows that $z \in \varrho_{\beta}$ for all $\beta \in\left(-K_{1}, \beta_{0}+K_{2}\right)$, in particular $z \in \varrho_{\beta_{0}}$. This proves

(ii) $\sigma_{\beta} \backslash \sigma_{\beta}^{r} \subset S_{\beta}(0)$.

Similarly, $\left\{\beta \in(-a, a) \mid \lambda \in \sigma_{d \beta}^{r}\right\}$ is open, and $\lambda \notin \sigma_{e \beta}$ for any $\beta \neq 0$ so the set $\sigma_{d \beta}^{r}$ is constant for $0<\beta<a$ ) and similarly for $-a<\beta<0$. Since a real eigenvalue of $H(\theta)$ is also an eigenvalue of $H(\bar{\theta})$, it follows that $\sigma_{d \beta}^{r}$ is constant for $0<|\beta|<a$. It follows immediately from (i) that $\sigma_{e \beta}^{r}=\{0\}$ for $0<|\beta|<a$, and $\sigma_{d \beta}^{r} \subset \Sigma_{\beta}^{r}=\{0\}$.

We finally prove that $\sigma_{\beta}^{r}$ is bounded and establish the estimate (iv).

Since $V$ is $H_{0}$-compact, it follows that $V$ is $H_{0}-\varepsilon$-bounded and hence $H=H_{0}+V$ is bounded below, so $\lambda_{0}=\inf _{\lambda \in \sigma} \lambda>-\infty$, and $\sigma_{0 d}$ is at most a bounded sequence of negative eigenvalues. By the above argument, these eigenvalues coincide with the negative eigenvalues of $H(\theta), 0<|\beta|<a$. Together with (ii) this implies that $\lambda_{0 \beta}^{r}=\lambda_{0}>-\infty$, and $\sigma_{\beta} \subset S_{\beta}\left(\lambda_{0}-1\right)$. Let $\varphi \neq-2 \beta$ be fixed, $\lambda=\varrho e^{i \varphi}, \varrho \in R^{+}$. Setting $K=\lambda_{0}-1$, and $\theta=i \beta$, we have

$$
H(i \beta)-K-\varrho e^{i \varphi}=e^{-2 i \beta} H_{0}-K-\varrho e^{i \varphi}+V(\theta) ;
$$

$\left\|\left(e^{-2 i \beta} H_{0}-K-\varrho e^{i \varphi}\right)^{-1}\right\|=\left\|\left(H_{0}-\left(K+\varrho e^{i \varphi}\right) e^{2 i \beta}\right)^{-1}\right\| \frac{1}{\mid \operatorname{Im}\left\{\left(K+\varrho e^{i \varphi}\right) e^{2 i \beta} \mid\right.}$

Hence for $f \in \mathscr{D}_{\boldsymbol{H}_{0}}$

$$
=\frac{1}{|K \sin 2 b+\varrho \sin (\varphi+2 \beta)|} \text {. }
$$

$$
\left\|\left(e^{-2 i \beta} H_{0}-K-\lambda\right) f\right\| \geqq|K \sin 2 \beta+\varrho \sin (\varphi+2 \beta)| \cdot\|f\| .
$$

The operators $V_{\alpha}(\theta)$ are $H_{0}^{\alpha}-\varepsilon$-bounded and hence $H_{0}-\varepsilon$-bounded, so $V(\theta)=\sum_{\alpha} V_{\alpha}(\theta)$ is $H_{0}-\varepsilon$-bounded, i.e.

$$
\|V(\theta) f\| \leqq \varepsilon\left\|e^{-2 i \beta} H_{0} f\right\|+K(\varepsilon)\|f\|, \quad f \in \mathscr{D}_{H_{0}} .
$$

This implies

$$
\|V(\theta) f\| \leqq \varepsilon\left\|\left(e^{-2 i \beta} H_{0}-K-\lambda\right) f\right\|+(\varepsilon|K+\lambda|+K(\varepsilon))\|f\| .
$$

(1) and (3) imply for $\varepsilon<1$

$$
\begin{aligned}
& \left\|\left(e^{-2 i \beta} H_{0}+V(\theta)-K-\lambda\right) f\right\| \geqq\{(1-\varepsilon) \cdot|K \sin 2 \beta+\varrho \sin (\varphi+2 \beta)| \\
& -\varepsilon|K+\lambda|-K(\varepsilon)\}\|f\| \text {. }
\end{aligned}
$$


Here $K$ and $b$ are fixed. Choose $\varepsilon$ such that $\varepsilon \leqq \frac{1}{2}(1-\varepsilon)|\sin (\varphi+2 \beta)|$. Then choose $\varrho_{0}$ such that

$$
\begin{gathered}
|K \sin 2 \beta| \leqq \frac{1}{2} \varrho_{0}|\sin (\varphi+2 \beta)|, \\
K \leqq \frac{1}{2} \varrho_{0}, K(\varepsilon) \leqq \frac{1}{8}(1-\varepsilon) \varrho_{0}|\sin (\varphi+2 \beta)| .
\end{gathered}
$$

Then (4) implies for $\varrho \geqq \varrho_{0}$

$$
\begin{aligned}
\|(H(i \beta)-K-\lambda) f\| & \geqq\left\{\frac{1}{2}(1-\varepsilon) \varrho|\sin (\varphi+2 \beta)|-\frac{1}{2} \varepsilon \varrho-K(\varepsilon)\right\}\|f\| . \\
& \geqq\left\{\frac{1}{4}(1-\varepsilon) \varrho|\sin (\varphi+2 \beta)|-K(\varepsilon)\right\}\|f\| \\
& \geqq \frac{1}{8}(1-\varepsilon) \varrho|\sin (\varphi+2 \beta)| \cdot\|f\| .
\end{aligned}
$$

(5) implies first of all, that there are no eigenvalues of $H(i \beta)$ on the ray $R_{\varphi}=\left\{\varrho e^{i \varphi} \mid 0 \leqq \varrho<\infty\right\}$ for $\varrho \geqq \varrho_{0}=\varrho_{0}(\varphi)$. In particular, the real eigenvalues of $H(\theta)$ and hence the eigenvalues of $H$ are bounded by $\varrho_{0}(\theta)$.

Moreover, for $|\varphi|>2 \beta$, the ray $R_{\varphi}$ is in the resolvent set of $H(i \beta)-K$, and since (5) is equivalent to

$$
\left\|\left(H(i b)-K-\varrho e^{i \varphi}\right)^{-1} g\right\| \leqq C_{1}(\varphi) \varrho^{-1}\|g\| \quad \text { for } \quad \varrho \geqq \varrho_{0}, \quad g \in \mathscr{H}
$$

with $C_{1}(\varphi)=\frac{8}{(1-\varepsilon)|\sin (\varphi+2 \beta)|}$ we obtain the estimate

$$
\left\|H(i \beta)-K-\varrho e^{i \varphi}\right\| \leqq C_{1}(\varphi) \varrho^{-1} \text { for } \varrho \geqq \varrho_{0} .
$$

Since $\left\|\left(H(i \beta)-K-\varrho e^{i \varphi} \|\right)^{-1}\right\|$ is continuous, it is bounded for $0 \leqq \varrho \leqq \varrho_{0}$, so there is a constant $C(\varphi)$, such that

$$
\left\|\left(H(i \beta)-K-\varrho e^{i \varphi}\right)^{-1}\right\| \leqq C(\varphi) \varrho^{-1} \text { for } 0 \leqq \varrho<\infty .
$$

2) Assume that (i)-(iv) hold true for all systems of $k \leqq n-1$ particles with $U(\theta)$-analytic relatively compact two-body interactions. We prove (i)-(iv) for any system of $n$ particles. We shall first prove

$$
\Sigma_{\beta}+e^{-2 \beta} R^{+} \cong \sigma_{e \beta} .
$$

According to our induction assumption it is sufficient to prove that for every decomposition $M=C_{1} \cup C_{2}$ one has

$$
\sigma\left(H^{C_{1}}(i \beta)\right)+\sigma\left(H^{C_{2}}(i \beta)\right)+e^{-2 i \beta} R^{+} C \sigma_{\beta} .
$$

Let $\lambda_{i} \in \sigma\left(H^{C_{\imath}}(i \beta)\right), k>0$ and $f_{i} \in \mathscr{D}\left(H^{C_{i}}(i \beta)\right), i=1,2$ such that

$$
\left\|f_{i}\right\|=1,\left\|\left(H^{C_{2}}(\theta)-\lambda_{i}\right) f_{i}\right\|<\varepsilon,
$$

and let $g \in \mathscr{D}\left(H_{0}^{\left(C_{1}, C_{2}\right)}\right)$ such that $\|g\|=1$ and $\left.\| H_{0}^{\left(C_{1}, C_{2}\right)}-k\right) g \|<\varepsilon$. This last property remains unchanged if $g$ is replaced by $g(\bar{a})$ obtained from $g$ by a 
translation $\bar{a}$ of the centers of mass of $C_{1}$ and $C_{2}$. From

one obtains

$$
H(i \beta)=H^{C_{1}}(i \beta)+H^{C_{2}}(i \beta)+e^{-2 i \beta} H_{0}^{\left(C_{1}, C_{2}\right)}+\sum_{\substack{\alpha=i j j) \\ i \in C_{1}, j \in C_{2}}} V_{\alpha}(i \beta)
$$

$$
\begin{aligned}
\|\left(H(i \beta)-\left(\lambda_{1}+\lambda_{2}\right.\right. & \left.\left.+e^{-2 i \beta} k\right)\right)\left(h_{1} \otimes h_{2} \otimes g(\bar{a})\right) \| \\
& <3 \varepsilon+\sum_{\substack{\alpha=(i j) \\
i \in C_{1}, j \in C_{2}}}\left\|V_{\alpha}(i \beta)\left(h_{1} \otimes h_{2} \otimes g(\bar{a})\right)\right\| .
\end{aligned}
$$

Now one has

$$
g(\bar{a})=e^{i \frac{m_{C_{1}} \bar{p}_{C_{2}}-m_{C_{2}} \bar{p}_{C_{1}}}{m_{C_{1}}+m_{C_{2}}} \bar{a}} g
$$

where $m_{c}$ and $\bar{p}_{c}$ are respectively the total mass and total momentum operator for cluster $C$

$$
\begin{aligned}
m_{c} & =\sum_{i \in C} m_{i} \\
\bar{p}_{c} & =\sum_{i \in C} \bar{p}_{i} .
\end{aligned}
$$

After some simple kinematical manipulations one gets

$$
e^{i \frac{m_{C_{1}} \bar{p}_{C_{2}}-m_{C_{2}} \bar{p}_{C_{1}}}{m_{C_{1}}+m_{C_{2}}}}=t_{\alpha}(\bar{a}) \otimes T_{\alpha}(\bar{a}), \lambda=(i, j), i \in C_{1}, j \in C_{2},
$$

where $t_{\alpha}(\bar{a})$ is a product of translation operators commuting with $V_{\alpha}$ and $T_{\alpha}$ is the representation of the translation group in $\mathscr{H}^{\alpha}$ given by

Accordingly

$$
T_{\alpha}(\bar{a})=e^{i \frac{m_{j} \bar{p}_{i}-m_{i} \bar{p}_{j}}{m_{i}+m_{j}} \bar{a}}
$$

$$
\left\|V_{\alpha}(i \beta) h_{1} \otimes h_{2} \otimes g(\bar{a})\right\|=\left\|V_{\alpha}(i \beta) T_{\alpha}(\bar{a}) h_{1} \otimes h_{2} \otimes g\right\| .
$$

Since $h_{1} \otimes h_{2} \otimes g \in \mathscr{D}\left(H_{0}\right) \subset \mathscr{D}\left(H_{0}^{\alpha}\right)$ we can rewrite this term as $\left\|A_{\alpha} T_{\alpha}(\bar{a}) \psi\right\|$ where $\psi=\left(H_{0}^{\alpha}-z\right)^{-1} h_{1} \otimes h_{2} \otimes g, \operatorname{Im} z \neq 0$, and $A_{\alpha}=V_{\alpha}(\mathrm{i} \beta)\left(H_{0}^{\alpha}-z\right)^{-1}$ is a compact operator on $\mathscr{H}^{\alpha}$. Now using (1) we can expand $\psi$ as an $l^{2}$-convergent sum $\psi=\sum_{n} h_{\alpha, n} \otimes \psi_{n}$ where

Then

$$
h_{\alpha, n} \in \mathscr{H}^{\alpha},\left\|h_{\alpha, n}\right\|^{2}=1 \quad \text { and } \quad\left(\psi_{n} \mid \psi_{m}\right)=\delta_{n m}\left\|\psi_{n}\right\|^{2} \text {. }
$$

$$
\left\|A_{\alpha} T_{\alpha}(\bar{a}) \psi\right\|^{2}=\operatorname{Trace}\left(B T_{\alpha}^{-1}(\bar{a}) A_{\alpha} T_{\alpha}(\bar{a})\right)
$$

where $B$ is the trace-class operator on $\mathscr{H}^{\alpha}$

$$
B=\sum_{n}\left\|\psi_{n}\right\|^{2}\left|h_{\alpha, n}\right\rangle\left\langle h_{\alpha, n}\right|, \quad \sum_{n}\left\|\psi_{n}\right\|^{2}=\|\psi\|^{2} .
$$


Since $A_{\alpha}$ is compact, $T_{\alpha}^{-1}(\vec{a}) A_{\alpha} T_{\alpha}(\bar{a})$ tends strongly to zero as $|\bar{a}| \rightarrow \infty$ which implies that the above trace tends to zero. From this we deduce that:

$$
\limsup _{|a| \rightarrow \infty}\left\|H(i \beta)-\left(\lambda_{1}+\lambda_{2}+e^{-2 i \beta} k\right)\left(h_{1} \otimes h_{2} \otimes g(a)\right)\right\|<3 \varepsilon .
$$

This implies that $\lambda_{1}+\lambda_{2}+e^{-2 i \beta} k \in \sigma_{\beta}$.

Now we prove that $\sigma_{\beta}$ is discrete outside $\Sigma_{\beta}+e^{-2 i \beta} R^{+}$, using the Weinberg equation.

We make use of the following theorem due to Ichinose [15]

Lemma 2. Let $A_{1}$ and $A_{2}$ be closed, densely defined operators in the separable Hilbert spaces $\mathscr{H}_{1}$ and $\mathscr{H}_{2}$, satisfying the conditions

$$
\begin{array}{ccc}
\sigma\left(A_{i}\right) \leqq\left\{z|| \arg z \mid \leqq \varphi_{i}\right\}, & \varphi_{i}, \varphi_{1}+\varphi_{2}<\pi, & i=1,2, \\
\left\|\left(A_{i}-\varrho e^{i \varphi}\right)^{-1}\right\| \leqq C(\varphi) \varrho^{-1} \text { for } \quad|\varphi|>\varphi_{i}, & i=1,2 .
\end{array}
$$

Then the operator $A=A_{1} \otimes I_{2}+I_{1} \otimes A_{2}$ is closable, and the spectrum of the closure $\tilde{A}$ is given by

$$
\sigma(\tilde{A})=\sigma\left(A_{1}\right)+\sigma\left(A_{2}\right) .
$$

Let $D_{k}$ be a decomposition of the system $M$ into $k$ disjoint clusters $C_{1}, \ldots, C_{k}$. Let

and

$$
\begin{aligned}
& V_{D_{k}}(\theta)=\sum_{\substack{i \in C_{i, j \in C_{j}} i \neq j \\
i \neq j}} V_{i j}(\theta), \\
& H_{D_{k}}(\theta)=H(\theta)-V_{D_{k}}(\theta)
\end{aligned}
$$

$$
R_{D_{k}}(\theta)=\left(H_{D_{k}}(\theta)-z\right)^{-1} \quad \text { for } \quad z \notin \sigma\left(H_{D_{k}}(\theta)\right) .
$$

We write $D_{k} \supset D_{l}$ if every cluster of $D_{k}$ is contained in a cluster of $D_{l}$. Let $S_{k}$ be a sequence of decompositions

$$
S_{k}=\left\{D_{n} \supset D_{n-1} \supset \cdots \supset D_{k}\right\}
$$

Set $V_{D_{l} D_{l-1}}(\theta)=V_{D_{l}}(\theta)-V_{D_{l-1}}(\theta)$ and

$$
\begin{gathered}
I(\theta, z)=\sum_{S_{2}} R_{D_{n}}(\theta, z) V_{D_{n} D_{n-1}}(\theta) \ldots R_{D_{2}}(\theta, z) V_{D_{2} D_{1}}(\theta), \\
D(\theta, z)=\sum_{S_{k}, k \geqq 2} R_{D_{n}}(\theta, z) V_{D_{n} D_{n-1}}(\theta) \ldots V_{D_{k+1} D_{k}}(\theta) R_{D_{k}}(\theta, z) .
\end{gathered}
$$

The Weinberg equations is derived from the Neumann series for the resolvent $R(\theta, z)$ in the same way as it was derived by Hunziker [9] for $\theta=0$. The equation is

$$
R(\theta, z)(1-I(\theta, z))=D(\theta, z),
$$


valid for all $z \in \varrho_{\beta}$ such that $I(\theta, z)$ and $D(\theta, z)$ are defined as bounded operators on $\mathscr{H}$. The Neumann series for $R(\theta, z)$ is

$R(\theta, z)=R_{0}(\theta, z)+\sum_{n=1}^{\infty} \sum_{\alpha_{1} \ldots \alpha_{n}} R_{0}(\theta, z) V_{\alpha_{1}} R_{0}(\theta, z) V_{\alpha_{2}}(\theta) \ldots V_{\alpha_{n}}(\theta) R_{0}(\theta, z)$

where $\alpha_{i}=\left(k_{i}, l_{i}\right), 0 \leqq k_{i}, l_{i} \leqq n, k_{i} \neq l_{i}$. From the $H_{0}(\theta)-\varepsilon$-boundedness of the operators $V_{\alpha}(\theta)$ and the fact that $\left\|R_{0}(\theta, z)\right\|=|z|^{-1}$ for

$$
\frac{\pi}{2}-2 \beta<\arg z<\frac{3 \pi}{2}-2 \beta
$$

it follows that the series (2) is norm-convergent for $\operatorname{Re}\left\{z e^{2 i \beta}\right\}<-K$, for some $K>0$. Then (1) is established as in [9] for $\operatorname{Re}\left\{z e^{2 i \beta}\right\}<-K$.

It follows from the induction assumption, that

and

$$
\sigma_{\beta}^{C} \subseteq S_{\beta}\left(\lambda_{0 \beta}^{C}-1\right)
$$

$$
\left\|\left(H^{C}(\theta)-\lambda_{0 \beta}^{C}+1-\varrho e^{i \varphi}\right)^{-1}\right\| \leqq C(\varphi) \varrho^{-1} \text { for }|\varphi|>2|\beta| .
$$

Applying Lemma 2 to the operators $H^{C}(\theta)-\lambda_{0 \beta}^{C}-1$ we obtain

$$
\sigma\left(H_{D_{k}}(\theta)\right)=\sigma_{\beta}^{C_{1}}+\cdots+\sigma_{\beta}^{C_{k}}+e^{-2 i \beta} R^{+} .
$$

Here we make use of the fact that

$$
\begin{gathered}
H_{D_{k}}(\theta)=\left(H^{C_{1}}(\theta) \otimes I^{C_{2}} \otimes \cdots \otimes I^{C_{k}}+\cdots+I^{C_{1}} \otimes \cdots \otimes H^{C_{k}}(\theta)\right) \otimes I^{\left\{C_{1} \ldots C_{k}\right\}} \\
+I^{C_{1}} \otimes \cdots \otimes I^{C_{k}} \otimes H_{0}^{\left\{C_{1} \ldots C_{k}\right\}}(\theta)
\end{gathered}
$$

where

$$
H^{C}(\theta)=e^{-2 \theta} H_{0}^{C}+\sum_{\substack{\alpha=(i, j) \\ \alpha \subset C}} V_{\alpha}(\theta)
$$

and $H_{0}^{\left(C_{1} \ldots C_{k}\right)}(\theta)=\mathrm{e}^{-2 \theta} H_{0}^{\left(C_{1} \ldots C_{k}\right)}$, where $H_{0}^{\left(C_{1} \ldots C_{k}\right)}$ is the Schrödinger operator with spectrum $[0, \infty)$ of the free relative morement of the centers of mass of $C_{1} \ldots C_{k}$.

From (3) and the previously proved inclusion it follows that

$$
\sigma\left(H_{D_{k}}(\theta)\right) \cong \sigma\left(H_{D_{l}}(\theta)\right) \text { for } D_{k} \supset D_{l} .
$$

Thus, all the resolvents occurring in the expressions of the operators $I(\theta, z)$ and $D(\theta, z)$ are analytic on the complement $G_{\beta}$ of the set

$$
\bigcup_{M=C_{1} \cup C_{2}}\left\{\sigma_{\beta}^{C_{1}}+\sigma_{\beta}^{C_{2}}+e^{-2 i \beta} R^{+}\right\}
$$

where we set $\sigma_{\beta}^{C}=\{0\}$ if $C$ contains only one particle.

Since the operators $V_{\alpha}(\theta)$ are $H_{0}(\theta)-\varepsilon$-bounded, and all the operators $H_{D_{k}}(\theta)$ have graph-norms equivalent to that of $H_{0}(\theta)$, it follows that $I(\theta, z)$, and $D(\theta, z)$ are analytic in the same domain $G_{\beta}$. From the induction 
assumption on the spectra $\sigma_{\beta}^{c}$ it follows that

$$
\bigcup_{M=C_{1} \cup C_{2}}\left\{\sigma_{\beta}^{C_{1}}+\sigma_{\beta}^{C_{2}}+e^{-2 i \beta} R^{+}\right\}=\Sigma_{\beta}+e^{-2 i \beta} R^{+} .
$$

It was proved by Combes [8] that $I(0, z)$ is compact for $\operatorname{Re} z<-K$. Choosing $K$ such that $\{z \mid \operatorname{Re} z<-K\} \subset G_{\beta}$ for all $\beta, a<\beta<a$, we conclude (see $[12,14]$ ) using the analyticity in $\theta$ of $I(\theta, z)$, that $I(\theta, z)$ is compact for all $z \in G_{\beta}$. Since $\|I(\theta, z)\| \underset{\operatorname{Re} z \rightarrow-\infty}{\longrightarrow} 0$, it follows from a result of Steinberg [12] that $(1-I(\theta, z))^{-1}$ is meromorphic for $z \in G_{\beta}$.

Hence

$$
R(\theta, z)=(1-I(\theta, z))^{-1} D(\theta, z)
$$

is meromorphic for $z \in G_{\beta}$, and $\sigma_{\beta} \cap G_{\beta}$ consists of isolated poles of $R(\theta, z)$.

Let $\lambda$ be an isolated pole of $R(\theta, z)$ and let $\Gamma$ be a closed circle with the usual orientation separating $\lambda$ from $\sigma_{\beta} \backslash\{\lambda\}$. Then the operator

$$
P_{\lambda}(\theta)=\frac{-1}{2 \pi i} \int_{\Gamma} R(\theta, z) d z
$$

is a projection on a closed subspace $\mathscr{H}_{\lambda}(\theta)$ of $\mathscr{H}$ such that $\mathscr{H}_{\lambda}(\theta)$ is invariant under $H(\theta)$ and $\sigma\left(H_{\lambda}(\theta)\right)=\{\lambda\}$, where $H_{\lambda}(\theta)=H(\theta) \mid \mathscr{H}_{\lambda}(\theta)$ (see [10], Ch. $10 \mathrm{III}, \S 6.5)$. Using (1) we have, by the analyticity of $D(\theta, z)$ in $G_{\beta}$,

$$
\begin{aligned}
P_{\lambda}(\theta) & =\frac{-1}{2 \pi i} \int_{\Gamma}(R(\theta, z)+D(\theta, z)) d z \\
& =\frac{1}{2 \pi i} \int_{\Gamma} R(\theta, z) I(\theta, z) d z
\end{aligned}
$$

The operator $R(\theta, z) I(\theta, z)$ is compact for $z \in G_{\beta}$, hence $P_{\lambda}(\theta)$, being the uniform limit of compact operators, is a compact projection, i.e. $\mathscr{H}_{\lambda}(\theta)$ is finite-dimensional. Thus, $\lambda$ is a finite-dimensional eigenvalue of $H(\theta)$.

We have proved that

(i) $\sigma_{e \beta}=\Sigma_{\beta}+e^{-2 i \beta} R^{+}$.

It follows immediately that $\sigma_{e \beta}^{r}=\Sigma_{\beta}^{r}$ is independent of $\beta, 0<|\beta|<a$ and $\sigma_{d_{\beta}}^{r \prime} \subset \Sigma_{\beta}^{r}$.

As for $n=2$ we prove, using the analyticity of the family $H(\theta)$, that $\sigma_{\beta} \mid \sigma_{\beta}^{r} \subset S_{\beta}\left(\lambda_{e \beta}\right)$ and that $\sigma_{d \beta}^{r}$ is independent of $\beta, 0<|\beta|<a$.

Then $C \backslash S_{\beta}\left(\lambda_{0 \beta}-1\right) \subset \varrho_{\beta}$, and we prove as for $n=2$, setting $K=\lambda_{0 \beta}-1$ that $\sigma_{\beta}^{r}$ is bounded and that the condition (iv) is satisfied. This concludes the proof of Lemma 1. 
We now make the connection with the spectrum $\sigma$ of $H$. First we prove

Lemma 3. The eigenvalues of $H$, which are not in $\Sigma \cap \sigma_{p}$ are precisely the real isolated eigenvalues of $H(\theta)$ outside $\Sigma, 0<|\beta|<$ a. i.e.

Proof. Let

$$
\sigma_{p} \backslash \Sigma=\sigma_{d \beta}^{r} \backslash \Sigma, \quad 0<|\beta|<a .
$$

$$
H=\int_{-\infty}^{\infty} \lambda d E_{\lambda}
$$

be the spectral resolution of the operator $H$. We have the following connection between $E_{\lambda}$ and $R(z)$ :

$$
E_{\lambda}-E_{\lambda-0}=\operatorname{sim}_{\substack{z \rightarrow \lambda \\ z \in C^{+}}}(z-\lambda) R(z) .
$$

Let $\mathscr{D}(\mathcal{O})$ be the set of vectors $\Phi$ in $\mathscr{H}$, such that the vector-valued function $\Phi(\theta)=U(\theta) \Phi$ has an analytic continuation to $\mathcal{O}$. The set $\mathscr{D}(\mathcal{O})$ is dense in $\mathscr{H}$ according to a theorem of Nelson [11] stating that $\mathscr{D}(C)$ is dense in $\mathscr{H}$.

For $\Phi \in \mathscr{D}(\mathcal{O})$ and $\theta \in R$ we have

$$
(\Phi, R(z) \Phi)=\left(\Phi, U(\theta)^{-1} R(\theta, z) U(\theta) \Phi\right)=(\Phi(\theta), R(\theta, z) \Phi(\theta)) .
$$

The function

$$
F_{\Phi}(\theta, z)=(\Phi(\bar{\theta}), R(\theta, z) \Phi(\theta))
$$

is for a fixed $z \in C^{+}=\{z \in C \mid \operatorname{Im} z>0\}$ analytic in $\theta$ for $\theta \in \mathcal{O}_{-\varepsilon(z)}^{+}$ $=\{\theta \in \mathcal{O} \mid \operatorname{Im} \theta>-\varepsilon(z)\}, \varepsilon(z)>0$, according to Lemma 1. Since $F_{\Phi}(\theta, z)$ $=(\Phi, R(z) \Phi)$ for $\theta \in R$, it follows that

$$
(\Phi, R(z) \Phi)=F_{\Phi}(\theta, z), \quad \theta \in \mathcal{O}_{-\varepsilon(z)}^{+} .
$$

Thus, (6) holds for any fixed $\theta \in \mathcal{O}^{+}$and all $z \in C^{+}$.

Lemma 1 implies that the function $F_{\Phi}(\theta, z)$ is meromorphic in $G_{\beta}$. Let $\lambda \in G_{\beta} \cap R=R \backslash \sum_{\beta}^{r}$. By (4) and (6)

$$
\left(\Phi,\left(E_{\lambda}-E_{\lambda-0}\right) \Phi\right)=\lim _{\substack{z \rightarrow \lambda \\ z \in C^{+}}}(z-\lambda)(\Phi, R(z) \Phi)=\lim _{\substack{z \rightarrow \lambda \\ z \in C^{+}}}(z-\lambda) F_{\Phi}(\theta, z) .
$$

It follows from (7), that

$$
\left(\Phi,\left(E_{\lambda}-E_{\lambda-0}\right) \Phi\right)=0 \quad \text { for all } \quad \Phi \in \mathscr{D}(\mathcal{O}) \quad \text { if } \quad \lambda \notin \sigma_{d \beta}^{r}
$$

and

$$
\left(\Phi,\left(E_{\lambda}-E_{\lambda-0}\right) \Phi\right) \neq 0 \text { for some } \quad \Phi \in \mathscr{D}(\mathcal{O}) \quad \text { if } \quad \lambda \in \sigma_{d \beta}^{r}
$$

since $\{\Phi(\theta) \mid \Phi \in \mathscr{D}(\mathcal{O})\}$ is dense in $\mathscr{H}$. 
Notice that if follows from (7), that the real poles of $R(\theta, z)$ are simple poles. This is not necessarily true for the poles in the interior of $S_{\beta}\left(\lambda_{e \beta}\right)$.

We now have all the tools required for the proof of our main results formulated in the following theorems.

Theorem 1. The point spectrum $\sigma_{p}$ of $H$ is bounded and the set $\sigma_{p}^{\prime}$ of its accumulation points is contained in the set of thresholds $\Sigma$.

The eigenspace corresponding to an eigenvalue $\lambda \notin \Sigma$ is finite dimensional, and bound-state wave functions are in $\mathscr{D}(\mathcal{O})$.

Proof. The first assertions follow immediately from Lemmas 1 and 3. In establishing the properties of the eigenspaces we follow the methods of Aguilar and Combes [7].

Let $\lambda \in \sigma_{p} \mid \Sigma$, then by Lemma $3, \lambda \in \sigma_{d \beta}^{r}$ for $0<|\beta|<a$. Let $P^{ \pm}(\theta, \lambda)$ be the projection on the eigenspace of $\mathscr{H}(\theta)$ corresponding to the eigenvalue $\lambda, \theta \in \mathcal{O}^{ \pm}$, defined by $P^{ \pm}(\theta, \lambda)=-\frac{1}{2 \pi i} \int_{\Gamma_{\theta}} R(\theta, z) d z$ where $\Gamma_{\theta} \cap \sigma_{\beta}=\{\lambda\}$. By [10], Ch. 7, $\S 3.1$, the operators $P^{ \pm}(\theta, \lambda)$ form analytic families of operators in $\mathcal{O}^{ \pm}=\{z \in \mathcal{O} \mid \operatorname{Im} z \gtrless 0\}$. We make use of the identity

to obtain

$$
\left(\Phi, E_{\lambda}-E_{\lambda-0} \Phi\right)=\lim _{\substack{z \rightarrow \lambda \\ z \in \hat{O}^{ \pm}}}(z-\lambda)(\Phi, R(z) \Phi)
$$

$$
\begin{aligned}
\left(\Phi, E_{\lambda}-E_{\lambda-0} \Phi\right) & =\lim _{\substack{z \rightarrow \lambda \\
z \in \mathcal{O}^{ \pm}}}(z-\lambda)(\Phi(\bar{\theta}) R(\theta, z) \Phi(\theta)) \\
& =\left(\Phi(\bar{\theta}), P^{ \pm}(\theta, \lambda) \Phi(\theta)\right) \text { for } \theta \in \mathcal{O}^{ \pm} .
\end{aligned}
$$

We now proceed as in [7] to prove, that

$$
P^{ \pm}(\theta, \lambda) \underset{\theta \rightarrow 0}{\longrightarrow} E_{\lambda}-E_{\lambda-0} \quad \text { uniformly }
$$

and moreover that the family of operators defined by

$$
P(\theta, \lambda)=\left\{\begin{array}{l}
P^{ \pm}(\theta, \lambda) \text { for } \theta \in \mathcal{O}^{ \pm} \\
U(\theta) E_{\lambda}-E_{\lambda-0} U(\theta)^{-1} \text { for } \quad \theta \in R
\end{array}\right.
$$

is analytic in $\mathcal{O}$. This implies, that $\lambda$ is a finite-dimensional eigenvalue of $H$, and that the corresponding eigenfunctions are in $\mathscr{D}(\mathcal{O})$.

Let us mention that if the interaction is local or electromagnetic, bound-state wave functions also satisfy strong decrease properties in configuration space. In fact using the analyticity property stated in Theorem 1 and arguments similar to those used in [9] (Theorem 4) one can show that if $\Phi$ is a bound-state wave-function corresponding to an 
eigenvalue $\lambda \notin \Sigma$, then $|(\Phi, Q(\vec{r}) \Phi)|<\infty$ for any polynomial $Q$. This kind of result will be investigated in more detail in a forthcoming paper.

Theorem 2. $\mathscr{H}=\mathscr{M}_{a}(H) \oplus \mathscr{M}_{d}(H)$. The spectrum of

$$
H_{a}=H \mid \mathscr{M}_{a} \text { is }\left[\lambda_{e}, \infty\right) \text {, where } \lambda_{e}=\min _{\lambda \in \Sigma} \lambda \text {. }
$$

Proof. Let us show that $\sigma_{s c}(H)=\emptyset$. Let $\Phi \in \mathscr{D}(\mathcal{O})$, and let $I=\left[\lambda_{1}, \lambda_{2}\right]$ be any closed interval on the real axis disjoint from $\sigma_{\beta}^{r}, \beta \neq 0$. Then by Lemma $2, I \cap \sigma_{p}=\emptyset$, and we have (see [10], VI, § 5.2)

$$
\begin{aligned}
\left(\Phi,\left(E_{\lambda_{2}}-E_{\lambda_{1}}\right) \Phi\right)= & \frac{1}{2 \pi i} \lim _{\varepsilon \rightarrow 0_{+}}\left\{\int_{\lambda_{1}-i \varepsilon}^{\lambda_{2}-i \varepsilon}(\Phi,(R(\lambda-i \varepsilon) \Phi) d \lambda\right. \\
& \left.-\int_{\lambda_{1}+i \varepsilon}^{\lambda_{2}+i \varepsilon}(\Phi, R(\lambda+i \varepsilon) \Phi) d \lambda\right\} .
\end{aligned}
$$

Since $C^{+} C \varrho_{\beta}$ for $\beta>0$ and $C^{-} \subset \varrho_{\beta}$ for $\beta<0$ we obtain as in the proof of Lemma 3 for a fixed $\theta \in \mathcal{O}^{-}$

$$
\begin{aligned}
\left(\Phi,\left(E_{\lambda_{2}}-E_{\lambda_{1}}\right) \Phi\right)= & \frac{1}{2 \pi i} \lim _{\varepsilon \rightarrow 0_{+}} \int_{\lambda_{1}}^{\lambda_{2}}\{(\Phi(\bar{\theta}), R(\theta, \lambda-i \varepsilon) \Phi(\theta)) \\
& -(\Phi(\theta), R(\bar{\theta}, \lambda+i \varepsilon) \Phi(\bar{\theta}))\} d \lambda \\
= & \frac{1}{2 \pi i} \int_{\lambda_{1}}^{\lambda_{2}}\{(\Phi(\bar{\theta}), R(\theta, \lambda) \Phi(\theta))-(\Phi(\bar{\theta}) R(\bar{\theta}, \lambda) \Phi(\bar{\theta}))\} d \lambda
\end{aligned}
$$

Since $I \subset \varrho_{\beta}, \quad \beta \neq 0$, and the functions $(\Phi(\bar{\theta}) R(\theta, \lambda-i \varepsilon) \Phi(\theta))$ and $(\Phi(\theta), R(\bar{\theta}, \lambda+i \varepsilon) \Phi(\bar{\theta}))$ have analytic continuations across $I$ from below and above respectively it follows that $\left(\Phi, E_{\lambda} \Phi\right)$ is absolutely continuous on $I$ for every $\Phi \in \mathscr{D}(\mathcal{O})$ and hence $\left\{E_{\lambda}\right\}$ is absolutely continuous on $I$.

Let $\Psi \in \mathscr{H}_{s c}$, then since $\mu\left(\operatorname{supp}\left(\Psi, E_{\lambda} \Psi\right)\right)=0$ the function $\left(\Psi, E_{\lambda} \Psi\right)$ is constant for $\lambda \in R \backslash \Sigma$, and it is clear from the continuity of $\left(\Psi, E_{\lambda} \Psi\right)$ and construction of $\Sigma$ that $\left(\Psi, E_{\lambda} \Psi\right)=$ constant $=0$ for all $\lambda$, i.e. $\Psi=0$, so $\mathscr{H}_{\text {sc }}=\emptyset$.

By Lemma $1, \sigma_{e}=\left[\lambda_{e}, \infty\right)$, and $\sigma_{p}^{\prime} \subset \Sigma$, so $\sigma\left(H_{a c}\right) \supseteqq\left[\lambda_{e}, \infty\right) \backslash \Sigma$, and hence $\left[\lambda_{e}, \infty\right) \supseteqq \sigma\left(H_{a c}\right) \supseteqq \overline{\left[\lambda_{e}, \infty\right) \backslash \Sigma}=\left[\lambda_{e}, \infty\right)$, so $\sigma\left(H_{a c}\right)=\left[\lambda_{e}, \infty\right)$.

This concludes the proof of Theorem 2 .

Acknowledgement. The authors are indebted to Dr. T. Ichinose, Nagoya University, for communicating the result quoted as Lemma 2.

The first author wishes to express his sincere thanks to Professor D. Kastler for his hospitality during the summer of 1970 , at the CPT, CNRS, Marseille where this research was initiated.

His work was supported in part by NSF grant GP-19651 and SUNY grant-in-aid 50-7163A. 


\section{References}

1. Hepp, K.: Helv. Phys. Acta 42, 425 (1969).

2. Lavine, R.: Commutations and scattering theory, Preprint, Cornell University (1970).

3. Combes, J.M.: Time dependent approach to non-relativistic multi-channel scattering. Nuovo Cimento 64, 111 (1969).

4. Dollard, J.: J. Math. Phys. 5, 729 (1964).

5. Buslaev,, Matreev,J.: Theoret. Math. 'Phys. (Russian), 1970.

6. Corbett, J. V.: Phys. Rev. 12, 333 (1970).

7. Aguilar,J., Combes, J. M.: A class of analytic perturbations for one-body Schrödinger Hamiltonians, Commun. math. Phys. 22, 269-279 (1971).

8. Combes, J.M.: Relatively compact interactions in many particle systems. Commun. Math. Phys. 12, 283 (1969).

9. Hunziker, W.: On the spectra of Schrödinger multiparticle Hamiltonians. Helv. Phys. Acta 39, 5, 451-462 (1966).

10. Kato, T.: Perturbation theory for linear operators. Berlin-Heidelberg-New York: Springer 1966.

11. Nelson, E.: Analytic vectors. Ann. Math. 70, 3 (1959).

12. Steinberg, S.: Arc. for Rat. Mechs. Anal. 31372 (1968).

13. Wolf, F.: On the essential spectrum of partial differential boundary problems. Commun. Pure Appl. Math. 12, 211-228 (1959).

14. Simon, B.: On the infinitude or finiteness of the number of bound states of an $n$-body quantum system, Preprint, Princeton University 1970.

15. Ichinose, T.: On operators on tensor products of Banach spaces, Preprint, Nagoya University.

J. M. Combes

Centre de Physique Théorique

C.N.R.S.

31 Chemin J. Aiguier

F-13 Marseille 9, France 